\title{
ANÁLISE DE PARTíCULAS MISTAS EM REJEITOS DE CONCENTRAÇÃO MAGNÉTICA
}

\author{
J. L. M. REIS ${ }^{{ }^{*}}$, C. A. PEREIRA', N. P. SAMPAIO ${ }^{2}$ \\ ${ }^{1}$ Universidade Federal de Ouro Preto (UFOP) - Departamento de Engenharia de Minas (DEMIN) \\ ${ }^{2}$ Universidade Federal de Ouro Preto (UFOP), Departamento de Engenharia de Materiais (REDEMAT) \\ jreis_lumia@hotmail.com
}

Submetido 21/12/2016 - Aceito 23/02/2017

DOI: $10.15628 /$ holos.2017.5481

\section{RESUMO}

Partículas mistas são partículas de diferentes minerais que estão intimamente associadas. Objetivou-se por meio deste estudo: (i) utilizar o MEV EDS na detecção de partículas mistas em rejeitos de concentração magnética úmida de alta intensidade em escala laboratorial; (ii) analisar a natureza dessas associações minerais (mineral-minério - mineral de ganga; mineral-minério mineral-minério) e (iii) avaliar a necessidade da promoção da liberação mineral nessas partículas no sentido de aumentar a recuperação metálica. Os parâmetros experimentais foram: aumento de 250x, tensão de aceleração $20 \mathrm{kV}$ e uso dos elétrons retroespalhados na obtenção das imagens. Os resultados mostraram que existem partículas mistas de mineral-minério - mineral de ganga e mineral-minério mineral-minério, e também que a liberação dessas partículas não é viável, devido ao tamanho em que se daria maior liberação. Conclui-se que esses dados podem auxiliar na definição de alternativa para aumentar a recuperação metálica nos materiais que geraram esses rejeitos.

PALAVRAS-CHAVE: partículas mistas, associações minerais, liberação mineral.

\section{ANALYSIS OF MIXED PARTICLES IN MAGNETIC CONCENTRATION TAILINGS}

\section{ABSTRACT}

Mixed particles are particles of different minerals that are closely associated. The objectives of this study were: (i) use the SEM EDXS in tailings mixed particle detection from wet high-intensity magnetic concentration in laboratory scale; (ii) analyze the nature of these mineral associations (mineral-ore -

gangue mineral; mineral-ore - mineral-ore) and (iii) assess the need of promotion of liberation in these mineral particles to increase metal recovery. The experimental parameters were: $250 x$ increase,
\end{abstract}

accelerating voltage of $20 \mathrm{kV}$ and use of backscattered electrons (BSE) in obtaining the images. The results showed that there are mixed particles of mineral-ore gangue mineral and mineral-ore - mineral- ore, and also that the liberation of these particles is not feasible, due to the size in which would give greater liberation. As conclusion these data can assist in defining an alternative to increase metal recovery in materials that have generated these tailings.

KEYWORDS: mixed particles, minerals associations, mineral liberation. 


\section{INTRODUÇÃO}

Partículas mistas são partículas de diferentes minerais que estão intimamente associadas, possuem contato físico. Segundo Cavalheiro (1984) é necessário aplicar a fragmentação no sentido de individualizar espécies que por gênese estão mecanicamente associadas, de forma a permitir que ocorra a separação física dessas fases minerais. Esse processo é conhecido como liberação. Kelly e Spottiswood (1982) distinguem a liberação intergranular como sendo aquela que ocorre nas fronteiras ou interfaces dos grãos (gerando partículas de apenas um mineral), enquanto a liberação transgranular ocorre através dos grãos e não promove a sua individualização e sim a geração de novas partículas mistas de menores dimensões. Os processos de cominuição acabam por gerar essas duas classes e o conhecimento do tamanho em que a liberação atinge porcentagem satisfatória para a eficiência dos processos de concentração é que definirá o grau de quebra necessário a ser aplicado nas partículas.

Wolff (2009) na tentativa de viabilizar o reaproveitamento de rejeitos com considerável teor de minério de ferro efetuou caracterizações físicas, químicas, mineralógicas e microestruturais desses materiais provenientes de sete usinas de processamento mineral. Caracterizações físicas, químicas, mineralógicas e microestruturais foram conduzidas no estudo de Castro (2012), que também trabalhou com lamas de minério de ferro e visava a produzir pellet feed partindo desse material. No estudo de Nascimento et al. (2014) rejeitos armazenados na barragem da Mina do Sossego foram caracterizados por difração de raios-X (DRX), microscopia eletrônica de varredura (MEV EDS) e espectroscopia Raman. Tonžetić et al. (2014) visando a gerar um modelo para indicar o grau de cominuição necessário para chegar ao tamanho de liberação de conglomerados e espessura/composição de bandas minerais em amostras de formações bandadas de ferro aplicaram o processamento de imagens na determinação da composição desses materiais. Quinteros et al. (2015) empregaram caracterizações mineralógicas, análise de tamanhos dos grãos, liberação e associações minerais para desenvolvimento de rotas de flotação para um minério de prata. Seu intuito era produzir pellet feed com rejeitos de processos de concentração de uma mineradora de ferro e obteve sucesso com a segunda rota de processo proposta. Paiva et al. (2015) também aplicaram caracterizações químicas, mineralógicas e microestruturais na avaliação da adequabilidade de rejeitos de minério de ferro em aplicação na produção de argamassas, concretos e cerâmicas. Guimarães et al. (2015) aplicaram o MEV em um estudo de liberação de concentrado e rejeito magnético e averiguaram que este parâmetro estava em torno de $71 \%$ para o ferro no rejeito e $82 \%$ para o ferro no concentrado. Através da revisão de literatura apresentada pode-se notar que estudos envolvendo o uso de MEV diretamente relacionados a estudos de liberação e também na identificação de minerais são comuns no setor mineral.

Os objetivos do estudo foram: (i) utilizar o MEV EDS na detecção de partículas mistas em rejeitos de concentração magnética úmida de alta intensidade em escala laboratorial; (ii) analisar a natureza dessas associações minerais (mineral-minério - mineral de ganga; mineral-minério mineral-minério) e (iii) avaliar a necessidade da promoção da liberação mineral nessas partículas no sentido de aumentar a recuperação metálica. 


\section{MATERIAIS E MÉTODOS}

Para esse estudo foram aplicados rejeitos de concentração magnética de alta intensidade desenvolvida em escala laboratorial. Os processos de preparação amostral e ensaios de concentração magnética são descritos em Reis et al. (2015).

Essas amostras foram encaminhadas para a preparação de seções polidas no Lamin (DEGEO UFOP). As seções polidas receberam um filme de carbono e foram analisadas por microscopia eletrônica de varredura (MEV) EDS no NanoLab (REDEMAT). Os parâmetros experimentais foram: aumento de 250x, tensão de aceleração 20 kV e uso dos elétrons retroespalhados na obtenção das imagens.

As imagens de campo luminoso virtual serviram para a visualização de partículas mistas. 0 Espectrômetro de Raios-X por Dispersão de Energia (EDS) auxiliou na análise química pontual para a identificação dos minerais e associações minerais. Com base na escala das imagens foram identificadas as dimensões aproximadas de inclusões minerais em partículas maiores para averiguação da necessidade e viabilidade da promoção da liberação nessas partículas mistas.

Foi desenvolvida uma análise do grau de liberação com o método de Gaudin através das partículas presentes nas imagens. Para obter esse índice as partículas totalmente livres multiplicadas pelo seu peso (Tabela 1) foram divididas pelo seu próprio somatório acrescidas das classes de partículas mistas (todas multiplicadas pelos seus respectivos pesos). O resultado foi multiplicado por 100 para que o índice fosse dado em porcentagem.

Tabela 1. Classes e pesos para análise do grau de liberação de Gaudin

\begin{tabular}{cccc}
\hline Classe & Identificador & Liberação [\%] & Peso [-] \\
\hline 1 & C1 & 0 a 10 & 0,05 \\
2 & C2 & 10 a 20 & 0,15 \\
3 & C3 & 20 a 30 & 0,25 \\
4 & C4 & 30 a 40 & 0,35 \\
5 & C5 & 40 a 50 & 0,45 \\
6 & C6 & 50 a 60 & 0,55 \\
7 & C7 & 60 a 70 & 0,65 \\
8 & C8 & 70 a 80 & 0,75 \\
9 & C9 & 80 a 90 & 0,85 \\
10 & C10 & 90 a 95 & 0,95 \\
Totalmente livre & T1 & 95 a 100 & 1,00 \\
\hline
\end{tabular}

Fonte: elaboração própria.

A análise de liberação por essa metodologia é proposta e aplicada no estudo de Henley (1983) e de Silva e Luz (2013). Vale ressaltar que ela nos apresenta estimativas semiquantitativas, isso por ser uma análise bidimensional de uma estrutura tridimensional. $O$ desvio padrão da reprodutibilidade dessa técnica é de aproximadamente 1,5 com 95\% de confiabilidade segundo King (1982 apud PORPHÍRIO et al., 2010). 


\section{RESULTADOS E DISCUSSÃO}

As Figuras 1 a 6 são correspondentes às imagens de MEV geradas para análise das partículas mistas presentes nos rejeitos de concentração magnética laboratorial:

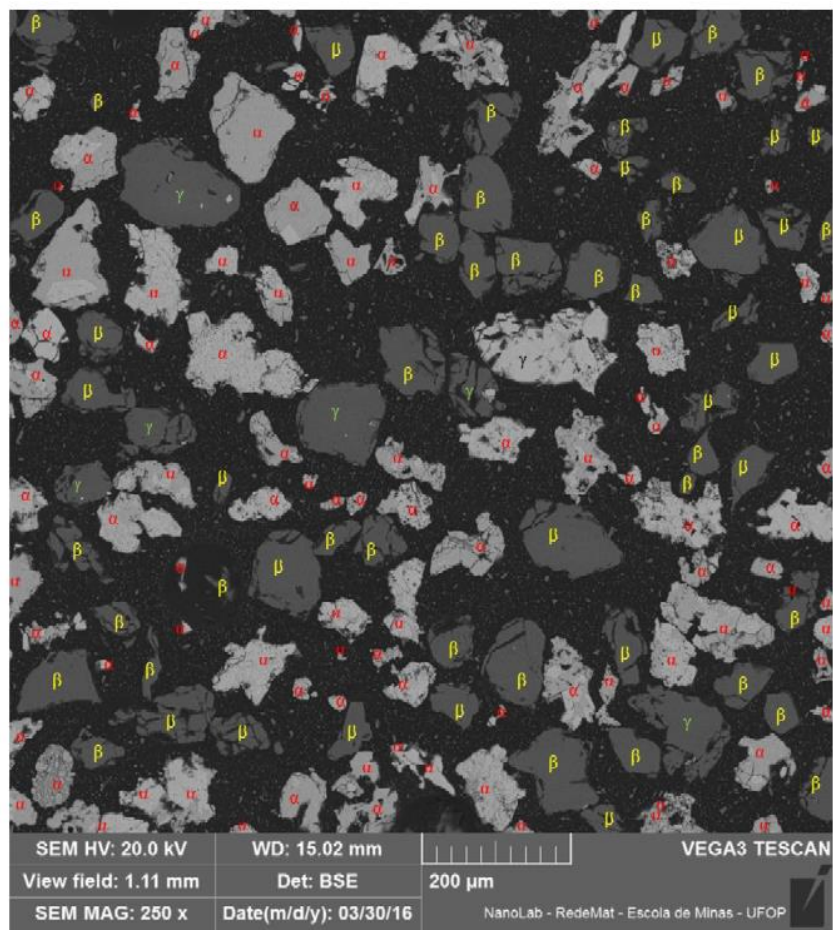

Figura 1. Mapeamento microestrutural da amostra do rejeito 1.

Observações: $\alpha$-partículas de Fe; $\beta$-partículas de ganga; $\boldsymbol{\gamma}$-partículas mistas. Fonte: análise de MEV EDS.

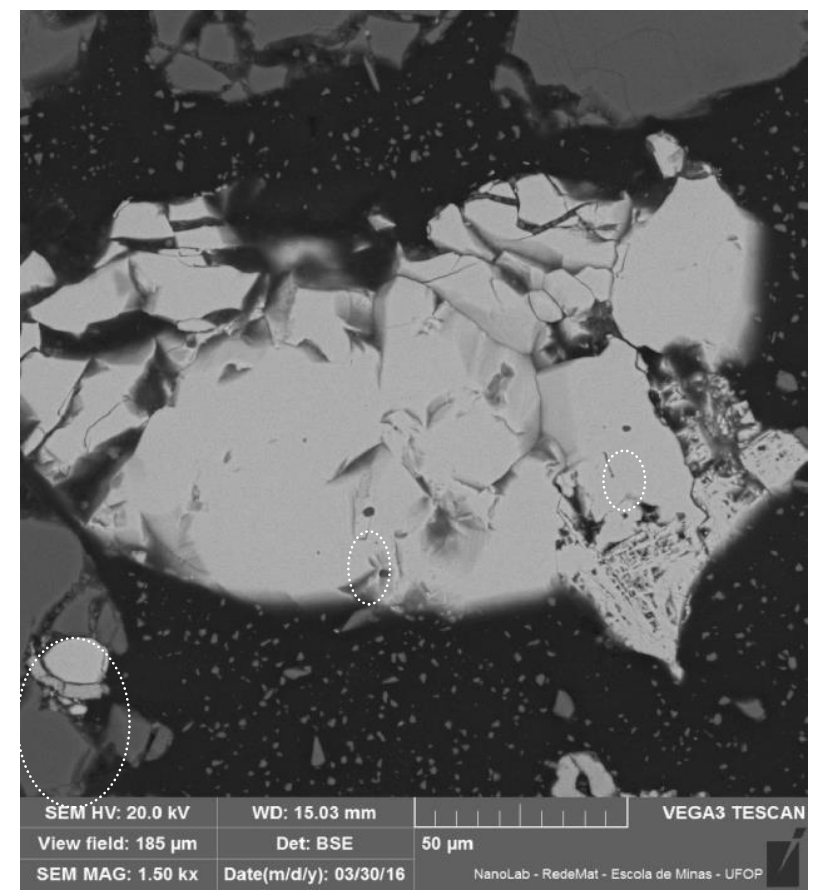

Figura 2. Detalhes de partículas mistas no rejeito 1.

Observações: aumento de 1500x. Fonte: análise de MEV EDS. 
No mapeamento microestrutural observado na Figura 1, a maioria das partículas mistas são partículas de quartzo com inclusões de minerais de ferro. Na Figura 2, percebem-se partículas mistas de minerais úteis (minerais de ferro - partículas mais claras) e minerais de ganga (quartzo - partículas mais escuras).

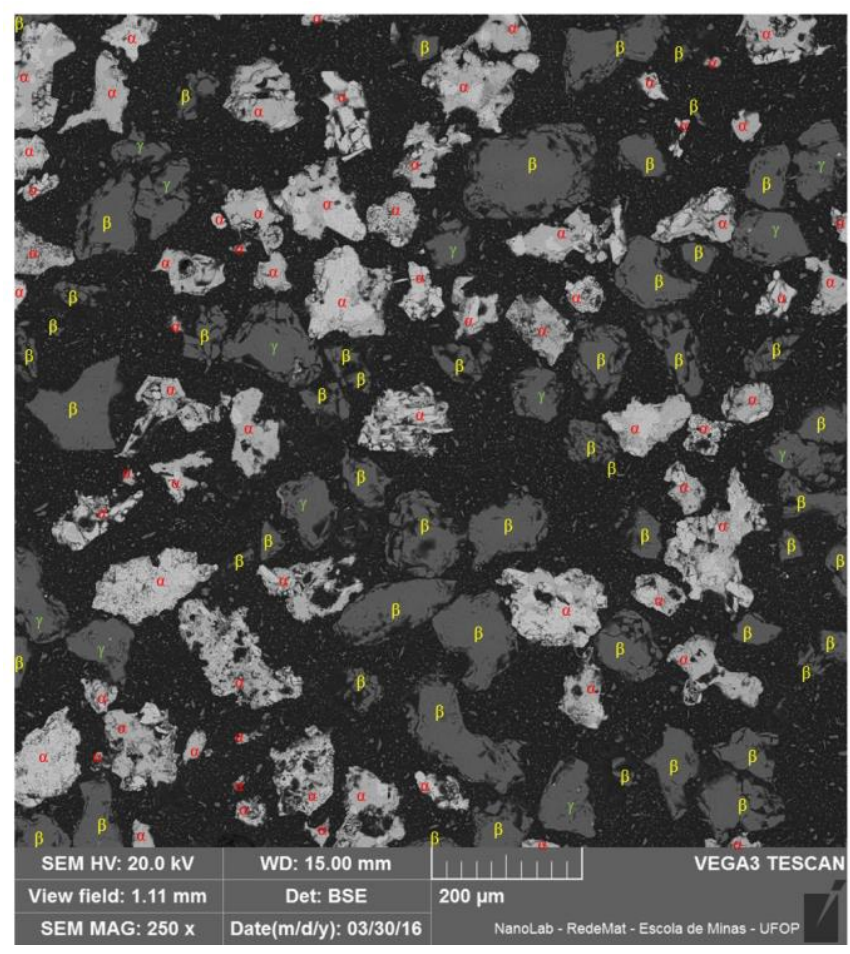

Figura 3. Mapeamento microestrutural da amostra do rejeito 2.

Observações: $\alpha$-partículas de Fe; $\beta$-partículas de ganga; $\gamma$-partículas mistas. Fonte: análise de MEV EDS.

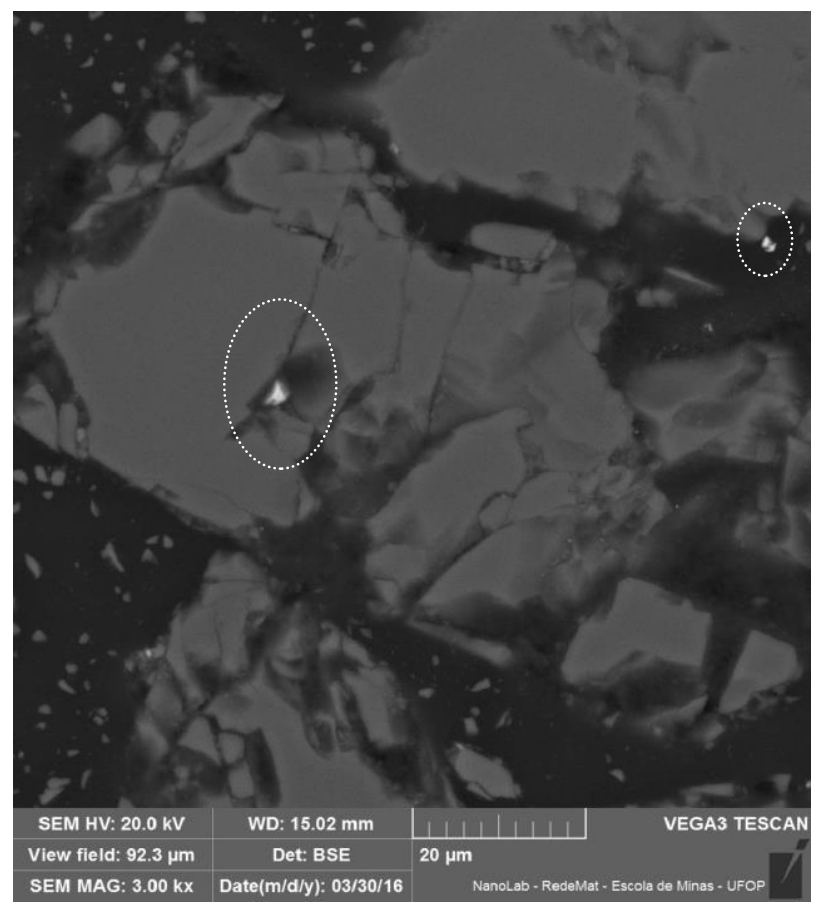

Figura 4. Detalhes de partículas mistas no rejeito 2.

Observações: aumento de 3000x. Fonte: análise de MEV EDS. 
A análise do mapeamento microestrutural observado na Figura 3 mostra que as partículas mistas presentes são partículas de quartzo com inclusões de minerais de ferro. Na Figura 4, detalhes desse tipo de partículas mistas são observáveis.

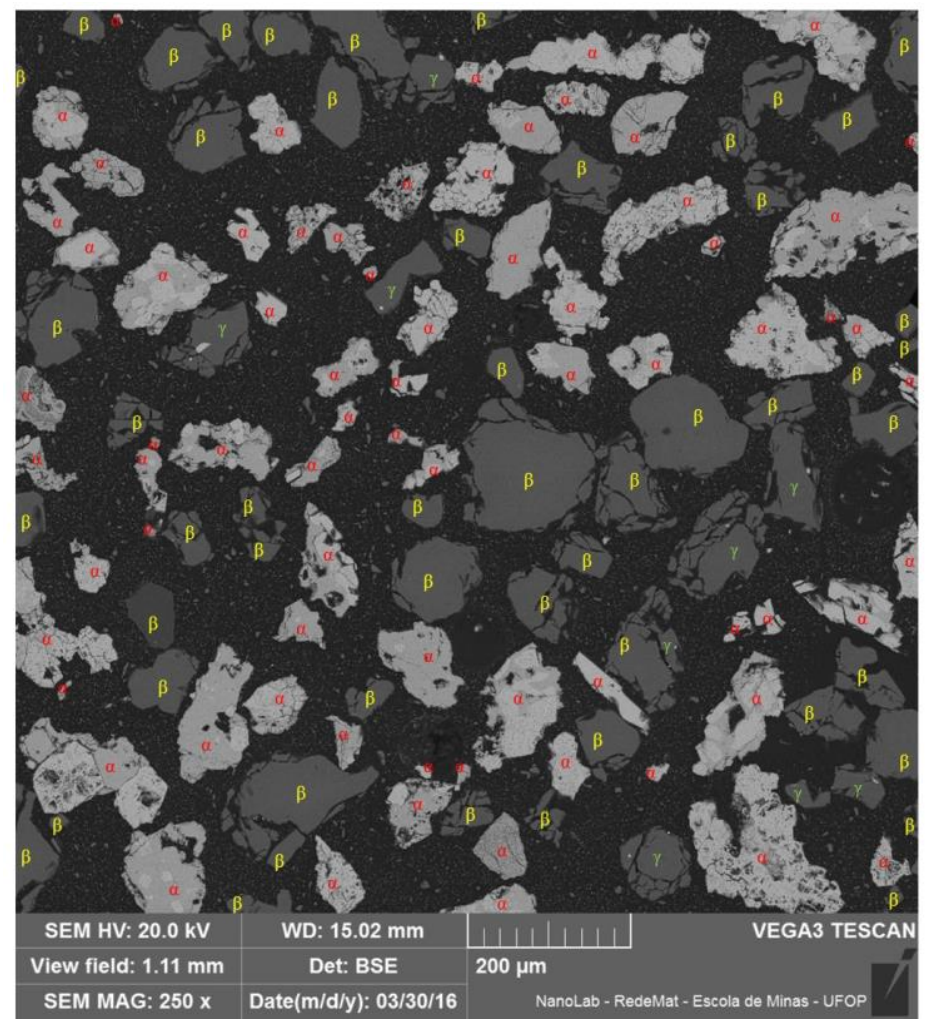

Figura 5. Mapeamento microestrutural da amostra do rejeito 3.

Observações: $\alpha$-partículas de Fe; $\beta$-partículas de ganga; $\gamma$-partículas mistas. Fonte: Análise de MEV EDS.
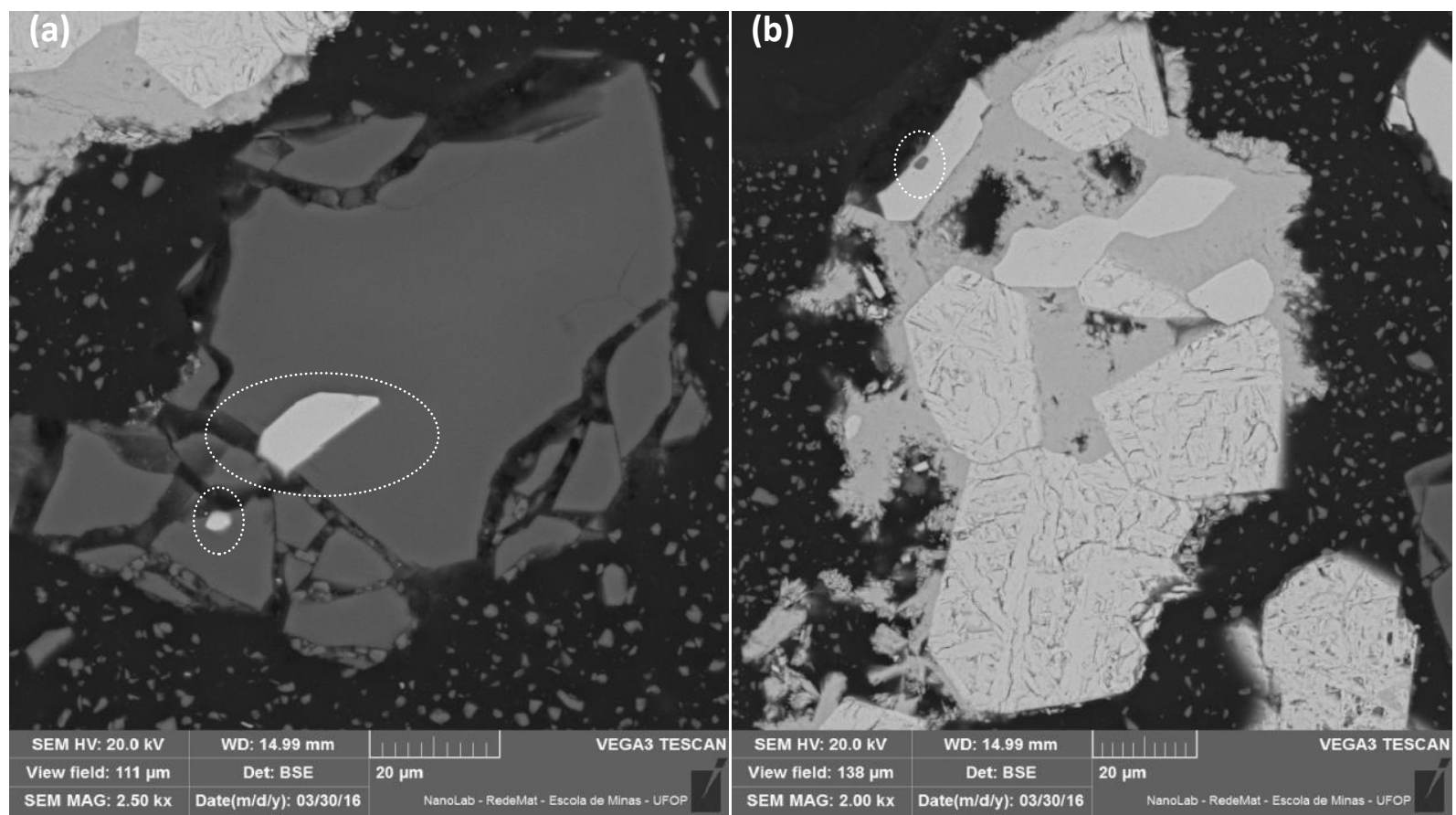

Figura 6. Detalhes de partículas mistas no rejeito 3.

Observações: aumentos de (a) 2500x e (b) 2000x. Fonte: análise de MEV EDS. 
As partículas mistas presentes no mapeamento microestrutural observado na Figura 5 são dos tipos quartzo com inclusões de minerais de ferro e, também, minerais de ferro com inclusões de quartzo. Partículas dessas duas naturezas são visualizadas na Figura 6.

Os tipos de associações minerais identificadas nas partículas mistas observadas nas amostras são: contato retilíneo; superfície conchoidal circundante e inclusões disseminadas.

Acerca dos mapeamentos microestruturais apresentados é importante ressaltar ainda que existem partículas mistas constituídas apenas de minerais de ferro. Essas associações minerais não foram tratadas nesse estudo como mistas, devido aos minerais que as constituem serem todos de interesse por se tratarem de minérios de ferro. Na Figura 6 (b), esse aspecto é mais nitidamente observável. Um espectro característico de uma partícula mista contendo magnetita e quartzo, proveniente do rejeito 2, é apresentado na Figura 7:

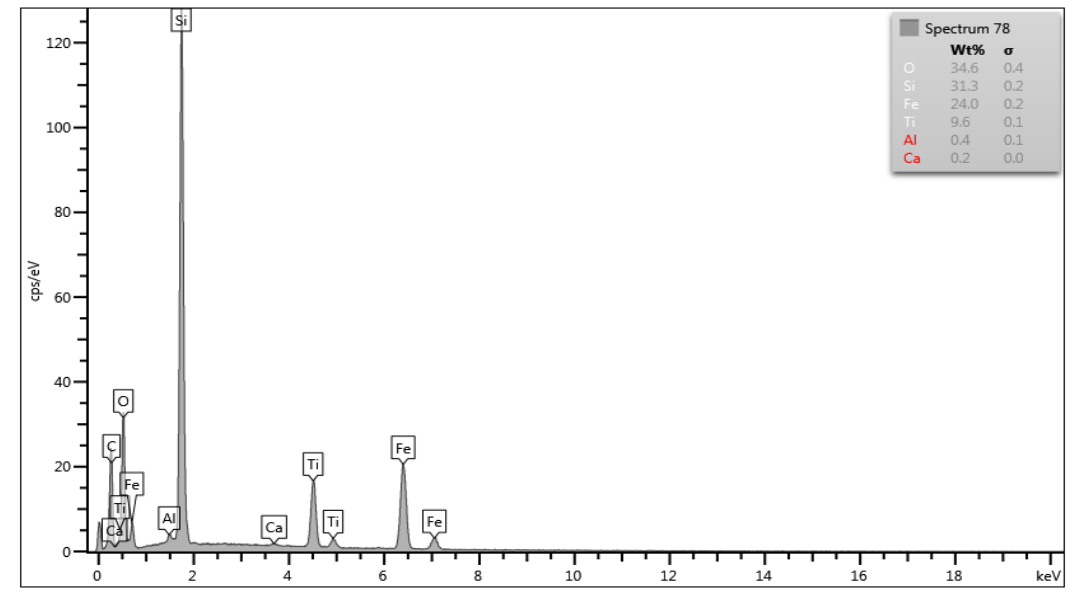

Figura 7. Espectro de partícula mista no rejeito 2. Fonte: análise de MEV EDS.

Na Figura 7, observam-se picos dos elementos O, Si, Fe, Ti e possível presença de Al e Ca em pequenas proporções.

Devido ao aumento adotado na microanálise (250x) não existem muitas partículas observáveis nas imagens, porém uma análise de liberação (Tabela 2) foi desenvolvida para estimar a liberação das partículas dos minerais de interesse e dos minerais de ganga.

Tabela 2. Estudo de liberação de rejeitos por análise de MEV

\begin{tabular}{|c|c|c|c|c|c|}
\hline \multirow{2}{*}{ Rejeito } & \multirow{2}{*}{ Partículas de Fe (TI) } & \multirow{2}{*}{ Partículas mistas } & \multirow{2}{*}{ Partículas de ganga (TI) } & \multicolumn{2}{|c|}{ Liberação } \\
\hline & & & & $\mathrm{Fe}[\%]$ & Ganga [\%] \\
\hline 1 & 103 & C1-1; C2-1; C10-5 & 56 & 95,41 & 91,88 \\
\hline 2 & 69 & $\mathrm{C} 9-2 ; \mathrm{C} 10-10$ & 52 & 86,03 & 82,28 \\
\hline 3 & 73 & C10-9 & 52 & 89,52 & 85,88 \\
\hline
\end{tabular}

Fonte: contagem de partículas em imagens de MEV.

Os dados apresentados na Tabela 2 evidenciam que a liberação dos minerais de interesse ainda está abaixo de $95 \%$ (exceto para o rejeito 1), que é o ponto ideal para uma melhor 
eficiência dos processos de concentração aplicáveis. Pode-se pensar que, partindo desse dado a decisão correta a ser tomada para que se possa aumentar a recuperação metálica é promover maior grau de cominuição às partículas visando a uma maior liberação. Porém, alguns aspectos devem ser analisados antes dessa decisão:

* Em qual faixa de tamanho essa liberação será atingida?

* Existem processos adequados para a concentração das partículas na dimensão de liberação requerida para 95\% de minerais úteis liberados?

* As diferenças de moabilidade entre minerais úteis e minerais de ganga são fator que pode levar a uma sobremoagem dos minerais úteis enquanto os minerais de ganga pouco sofreriam cominuição?

Essas questões estão entre as fundamentais no processo de tomada dessa decisão. Para efeito de uma análise simplificada no caso dos rejeitos em apreciação, as partículas mistas em consideração (minerais úteis inclusos em minerais de ganga) são de dimensões muito pequenas. A maior partícula mista encontrada tem diâmetro máximo de $20 \mu \mathrm{m}$ (na inclusão de minério de ferro) e a maioria dessas partículas está abaixo de $10 \mu \mathrm{m}$. Elas, caso fragmentadas para atingir liberação, entrariam em uma faixa de tamanho em que os processos de concentração já não apresentam eficiência considerável. Adotando a hematita e o quartzo para discutir sobre as diferenças de moabilidade, pode-se dizer que o mineral de ferro se fragmenta mais facilmente que o mineral de ganga - fator possivelmente relacionado às diferenças de dureza e sistemas cristalinos - e que esse fator pode levar a uma geração de lamas na moagem. Caso isso ocorresse, a tentativa de liberação apenas aumentaria o problema, pois as lamas geradas, ricas em minerais de ferro, se somariam ao fluxo de rejeitos e reduziriam a recuperação metálica.

Com esses dados foi possível constatar que, devido ao tamanho diminuto das partículas de interesse associadas aos minerais de ganga, não é viável tecnicamente, economicamente e ambientalmente promover a liberação dessas partículas para aumentar a recuperação metálica. O fator técnico relacionado à grande necessidade de aplicação de cominuição para atingir $95 \%$ de liberação. O ponto de vista econômico encontra limitações no grande aumento de consumo na energia para elevar a liberação desse material e o pouco incremento na receita que seria promovido com essas partículas livres. Ambientalmente destaca-se o fato da provável produção de lamas na moagem do material, fato que levaria a maior produção de rejeitos. Isso ainda levaria a mais implicações econômicas: maior necessidade de área para disposição desses efluentes e mais perda metálica por ineficiência de processos em granulometrias tão pequenas.

\section{CONCLUSÕES}

O estudo permitiu a detecção da presença de partículas mistas nos rejeitos de concentração magnética estudados, através de imagens de MEV. Essas imagens permitiram, com o auxílio do EDS, identificar a natureza dessas associações minerais (mineral-minério - mineral de ganga; mineral-minério - mineral-minério).

Conclui-se que os objetivos propostos para esse estudo puderam ser alcançados e que esses dados podem auxiliar na escolha de diferente alternativa para elevar a recuperação metálica do material gerador desses rejeitos. 


\section{AGRADECIMENTOS}

Os autores agradecem ao Marco Silva do Lamin DEGEO UFOP pela preparação das seções polidas e ao NanoLab REDEMAT UFOP pelas análises de MEV EDS.

\section{REFERÊNCIAS}

Castro, E. F. (2012). Produção de pellet feed a partir de lamas. Dissertação de mestrado, Universidade Federal de Minas Gerais, Belo Horizonte, MG, Brasil.

Cavalheiro, A. A. T. (1984). Libertação de minérios por cominuição. Porto: Demin FEUP.

Guimarães, F. A. V.; Barbosa, M. G.; Araújo, A. C.; Lacoste, M. (2015, outubro). Estudo da liberação do minério magnético de Peña Colorada no México. Anais do ENTMME - Encontro Nacional de Tratamento de Minérios e Metalurgia Extrativa, Poços de Caldas, MG, Brasil, 26.

Henley, K. J. (1983). Ore dressing mineralogy - a review of techniques, applications and recent developments. Geological Society of South Africa. Special publication. 7, 175-200.

Kelly, E. G.; Spottiswood, D. J. (1982). Introduction to mineral processing. New York: John Wiley \& Sons.

Nascimento, J. C.; Cordeiro, H. C.; Coral, N. F. A. F.; Corrêa, J. A. M.; Moreira, S. G. C.; Rodrigues, E. M. S. (2014, outubro). Caracterização de resíduos sólidos da barragem de rejeitos das Minas do Sossego (Canaã dos Carajás-PA). Anais do COBEQ - Congresso Brasileiro de Engenharia Química, Florianópolis, SC, Brasil, 20.

Paiva, P. R. P.; Silva, V. C.; Marques, A. R.; Ferreira, A. M. (2015, outubro). Caracterização química e mineralógica de resíduos de minério de ferro utilizando o Método Rietveld. Anais do ENTMME - Encontro Nacional de Tratamento de Minérios e Metalurgia Extrativa, Poços de Caldas, MG, Brasil, 26.

Porphírio, N. H. (in memorian); Barbosa, M. I. M.; Bertolino, L. C. (2010). Caracterização mineralógica de minérios: parte I. In: Da Luz, A. B.; Sampaio, J. A.; França, S. C. A. Tratamento de minérios (5. ed., Cap. 3., p. 55-84). Rio de Janeiro: CETEM/MCT.

Quinteros, J.; Wightman, E.; Johnson, N. W.; Bradshaw, D. (2015). Evaluation of the response of valuable and gangue minerals on a recovery, size and liberation basis for a low-grade silver ore. Minerals Engineering, 74, 150-155.

Reis, J. L. M.; Rafael, G. S.; Dias, A. C.; Pereira, C. A. (2015, outubro). Concentração magnética de materiais goethíticos do Quadrilátero Ferrífero Anais do ENTMME - Encontro Nacional de Tratamento de Minérios e Metalurgia Extrativa, Poços de Caldas, MG, Brasil, 26.

Silva, M. B.; Luz, J. A. M. (2013). Concentração magnética esgotadora de ultrafinos itabiríticos. REM: R. Esc. Minas, 66 (4), 499-505.

Tonžetić, I.; Duncan, M.; Bramdeo, S. (2014). The autosem ore characterisation of conglomeratic and banded iron formations. Minerals Engineering, 61, 54-65.

Wolff, A. P. (2009). Caracterização de rejeitos de minério de ferro de minas da Vale, Dissertação de mestrado, Universidade Federal de Ouro Preto, Ouro Preto, MG, Brasil. 